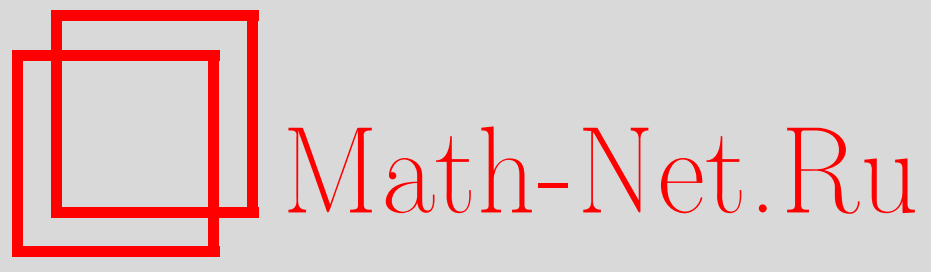

А. В. Фаминский, Р. В. Кувшинов, Начально-краевые задачи для обобщенного уравнения Кавахары, УМH, 2011, том 66, выпуск 4, 187-188

DOI: https://doi.org/10.4213/rm9427

Использование Общероссийского математического портала Math-Net.Ru подразумевает, что вы прочитали и согласны с пользовательским соглашением http://www . mathnet.ru/rus/agreement

Параметры загрузки:

IP : 54.162 .27 .143

26 апреля 2023 г., 14:24:03

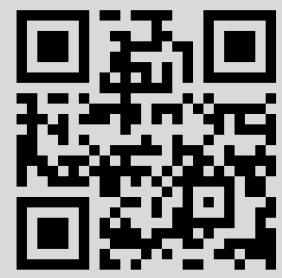




\section{Начально-краевые задачи для обобщенного уравнения Кавахары}

\section{А. В. Фаминский, Р. В. Кувшинов}

Для обобщенного уравнения Кавахары

$$
u_{t}-u_{x x x x x}+b u_{x x x}+a u_{x}+g(u) u_{x}=f(t, x)
$$

$(u=u(t, x), a, b \in \mathbb{R})$ рассматриваются две начально-краевые задачи: 1$)$ в полуполосе $\Pi_{T}^{+}=(0, T) \times \mathbb{R}_{+}\left(\mathbb{R}_{+}=(0,+\infty)\right)$ и 2$)$ в ограниченном прямоугольнике $Q_{T}=(0, T) \times$ $(0,1)$. Для обеих задач ставятся начальное условие

$$
u(0, x)=u_{0}(x), \quad x \geqslant 0 \quad \text { или } \quad x \in[0,1],
$$

и краевые условия на левой границе $(t \in[0, T])$

$$
u(t, 0)=\mu_{0}(t), \quad u_{x}(t, 0)=\mu_{1}(t),
$$

а для второй задачи ставятся также краевые условия на правой границе

$$
u(t, 1)=\nu_{0}(t), \quad u_{x}(t, 1)=\nu_{1}(t), \quad u_{x x}(t, 1)=\nu_{2}(t) .
$$

Предполагается, что функция $g$ имеет не более чем линейный порядок роста на бесконечности, а именно, для некоторой константы $c$

$$
\left|g^{\prime}(u)\right| \leqslant c \quad \forall u \in \mathbb{R}, \quad g(0)=0 .
$$

Устанавливаются результаты о глобальной корректности указанных задач $(T>0-$ произвольно) в классах как обобщенных, так и гладких решений.

Уравнение (1) при $g(u) \equiv u$ носит название уравнения Кавахары и описывает распространение длинных нелинейных волн в средах со слабой дисперсией (см., например, [1]). Последние результаты о глобальной корректности задачи Коши для этого уравнения можно найти в [2]. В случае нулевых краевых функций (и правой части $f$ ) глобальная корректность рассматриваемых задач для уравнения Кавахары установлена в [3] и [4]. Первые результаты в случае неоднородных краевых условий для задачи в $\Pi_{T}^{+}$для уравнения (1) были получены в [5] и [6].

Символ $I$ в дальнейшем обозначает или полуось $\mathbb{R}_{+}$или интервал $(0,1)$, a $k, l$, $m$ - целые неотрицательные числа. Решения рассматриваемых задач строятся в пространствах $X_{k}((0, T) \times I)$ функций $u(t, x)$ таких, что

$$
\begin{aligned}
\partial_{t}^{m} u & \in C\left([0, T] ; H^{k-5 m}(I)\right), \quad 5 m \leqslant k, \\
\partial_{x}^{l} u & \in C_{\mathrm{b}}\left(\bar{I} ; H^{(k-l+2) / 5}(0, T)\right), \quad l \leqslant k+2,
\end{aligned}
$$

и в случае задачи в $\Pi_{T}^{+}$дополнительно

$$
\partial_{t}^{m} \partial_{x}^{l} u \in L_{8}\left(0, T ; C_{\mathrm{b}}\left(\overline{\mathbb{R}}_{+}\right)\right), \quad 5 m+l \leqslant k,
$$

и при $k \geqslant 2$

$$
\partial_{t}^{m} \partial_{x}^{l} u \in L_{2}\left(\mathbb{R}_{+} ; C[0, T]\right), \quad 5 m+l \leqslant k-2
$$

(для задачи в $Q_{T}$ аналогичные свойства следуют из первых двух; символ $C_{\mathrm{b}}$ обозначает пространство непрерывных и ограниченных функций).

Для описания свойств правой части уравнения введем пространство

$$
M_{k}((0, T) \times I)=\left\{f: \partial_{t}^{m} f \in L_{2}\left(0, T ; H^{k-5 m}(I)\right), m \leqslant \frac{k+2}{5}\right\} .
$$


Кроме того, для определения условий согласования граничных данных, введем индуктивным способом специальные функции $\Phi_{m}(x)$, а именно, положим $\Phi_{0} \equiv u_{0}$ и для любого натурального $m$

$$
\left.\Phi_{m} \equiv \partial_{t}^{m-1} f\right|_{t=0}+\Phi_{m-1}^{(5)}-b \Phi_{m-1}^{\prime \prime \prime}-a \Phi_{m-1}^{\prime}-\left.\partial_{t}^{m-1}\left[g(u) u_{x}\right]\right|_{\partial_{t}^{l} \partial_{x}^{j} u=\Phi_{l}^{(j)}, l \leqslant m-1} .
$$

Tеорема 1. Пусть $g \in C^{k+1}(\mathbb{R})$, выполнено условие (5), $u_{0} \in H^{k}\left(\mathbb{R}_{+}\right), \mu_{0} \in$ $H^{(k+2) / 5}(0, T), \mu_{1} \in H^{(k+1) / 5}(0, T), f \in M_{k}\left(\Pi_{T}^{+}\right) \partial \Omega \Omega k \geqslant 2, \mu_{0}^{(m)}(0)=\Phi_{m}(0) n p u$ $m<k / 5, \mu_{1}^{(m)}(0)=\Phi_{m}^{\prime}(0)$ nри $m<(k-1) / 5$. Тогда задача (1)-(3) корректна в пространстве $X_{k}\left(\Pi_{T}^{+}\right)$.

Tеорема 2. Пусть $g \in C^{k+1}(\mathbb{R})$, выполнено условие (5), $u_{0} \in H^{k}(0,1), \mu_{0}, \nu_{0} \in$ $H^{(k+2) / 5}(0, T), \mu_{1}, \nu_{1} \in H^{(k+1) / 5}(0, T), \nu_{2} \in H^{k / 5}(0, T), f \in M_{k}\left(Q_{T}\right)$ для $k \geqslant 0$, $\mu_{0}^{(m)}(0)=\Phi_{m}(0), \nu_{0}^{(m)}(0)=\Phi_{m}(1)$ npu $m<k / 5, \mu_{1}^{(m)}(0)=\Phi_{m}^{\prime}(0), \nu_{1}^{(m)}(0)=\Phi_{m}^{\prime}(1)$ при $m<(k-1) / 5, \nu_{2}^{(m)}(0)=\Phi_{m}^{\prime \prime}(1)$ nри $m<(k-2) / 5$. Тогда задача (1)-(4) корректна в пространстве $X_{k}\left(Q_{T}\right)$.

Доказательство этих результатов для случая самого уравнения Кавахары можно найти в [7]-[9].

Под корректностью понимается, что решение в указанном пространстве существует, единственно и непрерывно зависит от начальной и краевых функций, а также от правой части уравнения.

Условия гладкости граничных функций являются естественными, поскольку для решения $v(t, x)$ из пространства $C_{\mathrm{b}}\left(\mathbb{R}^{t} ; H^{k}(\mathbb{R})\right)$ линейной задачи Коши

$$
v_{t}-v_{x x x x x}=0, \quad v(0, x)=v_{0}(x) \in H^{k}(\mathbb{R}),
$$

справедливы соотношения

$$
\left\|D_{t}^{2 / 5} v(\cdot, x)\right\|_{H^{k / 5}\left(\mathbb{R}^{t}\right)}=\left\|D_{t}^{1 / 5} v_{x}(\cdot, x)\right\|_{H^{k / 5}\left(\mathbb{R}^{t}\right)}=\left\|v_{x x}(\cdot, x)\right\|_{H^{k / 5}\left(\mathbb{R}^{t}\right)} \sim\left\|v_{0}\right\|_{H^{k}(\mathbb{R})} .
$$

\section{Список литературы}

[1] T. Kawahara, J. Phys. Soc. Japan, 33:1 (1972), 260-264. [2] Hua Wang, Shang Bin Cui, Dong Gao Deng, Acta Math. Sinica (Engl. Ser.), 23:8 (2007), 1435-1446. [3] N. A. Larkin, G. G. Doronin, Bol. Soc. Parana. Mat. (3), 25:1-2 (2007), 9-16. [4] G. G. Doronin, N. A. Larkin, Discrete Contin. Dyn. Syst. Ser. B, 10:4 (2008), 783-799. [5] К. Сангаре, Вестн. РУДН. Сер. матем., 10:1 (2003), 91-107. [6] К. Сангаре, А. В. Фаминский, Матем. заметки, 85:1 (2009), 98-109; англ. пер.: K. Sangare, A. V. Faminskii, Math. Notes, 85:1 (2009), 90-100. [7] Р. В. Кувшинов, А. В. Фаминский, Дифференц. уравнения, 45:3 (2009), 391-402; англ. пер.: R. V. Kuvshinov, A. V. Faminskii, Differ. Equ., 45:3 (2009), 404-415. [8] Р. В. Кувшинов, Вестн. РУДН. Сер. матем., инф., физ., 3 (2010), 5-16. [9] Р. В. Кувшинов, Вестн. РУДН. Сер. матем., инф., физ., 4 (2010), 35-47.

\section{A. В. Фаминский (A. V. Faminskii)}

Российский университет дружбы народов

E-mail: afaminskii@sci.pfu.edu.ru

\section{P. В. Кувшинов (R. V. Kuvshinov)}

Российский университет дружбы народов

E-mail: infty@mail.ru
Представлено М. И. Зеликиным Принято редколлегией 25.04.2011 